\title{
SUR LA RẼCOLTE DANS LE SUD-OUEST DE LA FRANCE \\ DE MOINA DUBIA DE GUERNe ET RICHARD \\ [Cladocères, Daphniidae]
}

$\operatorname{par} \mathbf{M}^{\mathrm{me}}$ J. REY.

L'examen d'un prélèvement effectué dans le lac de Rabastens le 24 août 1964, nous a révélé la présence d'une population de Moina dont les individus ne s'identifiaient à aucune des trois espèces du genre connues en France : Moina rectirostris (LEYDIG), Moina brachiata (Jurine), Moina macrocopa Straus.

L’étude systématique du matériel récolté nous a permis de rapporter cette espèce à Moina dubia, décrite du Sénégal par de Guerne et Richard [1892]. Moina dubia est une espèce à répartition essentiellement équatoriale, tropicale et subtropicale. Depuis le type récolté à Rufisque (Sénégal), elle a été retrouvée à plusieurs reprises en Afrique [W. 'Weltaker 1898, S. Fkman 1904, E. Daday 1910, R. Gurney 1911, A. Steuer 1939, P. M. Jenkin 1934, H. Gauthier 1937, 1939, 1955, J. P. Harding 1957, H. Löffler 1963], au Vénézuela [W. Ramner 1933, R. Margalef 1961], Queensland [R. GurNey 1927], Java [W. Ramner 1937], Formose, Mongolie, Mandchourie [M. Ueno 1938 a, 1938 b, 1939], Iran [H. LöfFler 1961]. En 1931, W. Ramner récoltait en Allemagne, dans l'Auensee près de Leipzig, une forme qu'il dénommait Moina dubia lacustris. C'est, à notre connaissance, la seule mention de $M$. dubia en Europe occidentale. La récolte d'une espèce de ce groupe dans le sud-ouest présente donc, outre le fait qu'elle est nouvelle pour la faune française, un intérêt particulier du point de vue biogéographique.

La station d'où proviennent les échantillons est un lac artificiel situé dans le Tarn, à proximité de la ville de Rabastens dont il assure l'alimentation en eau potable. Ses caractéristiques sont les suivantes : superficie, 6 ha environ; profondeur maxima, $6,40 \mathrm{~m}$; fond essentiellement argileux ; rives pourvues d'une abondante végétation phanérogamique; température de l'eau (relevée depuis la berge) à la date du prélèvement, $26^{\circ} \mathrm{C}$.

Moina dubia a été recueillie dans une zone à Chara - Typha Mentha, associée à Simocephalus vetulus, Sida crystallina, Scapholeberis mucronata, Chydorus sphaericus. Le matériel récolté com- 


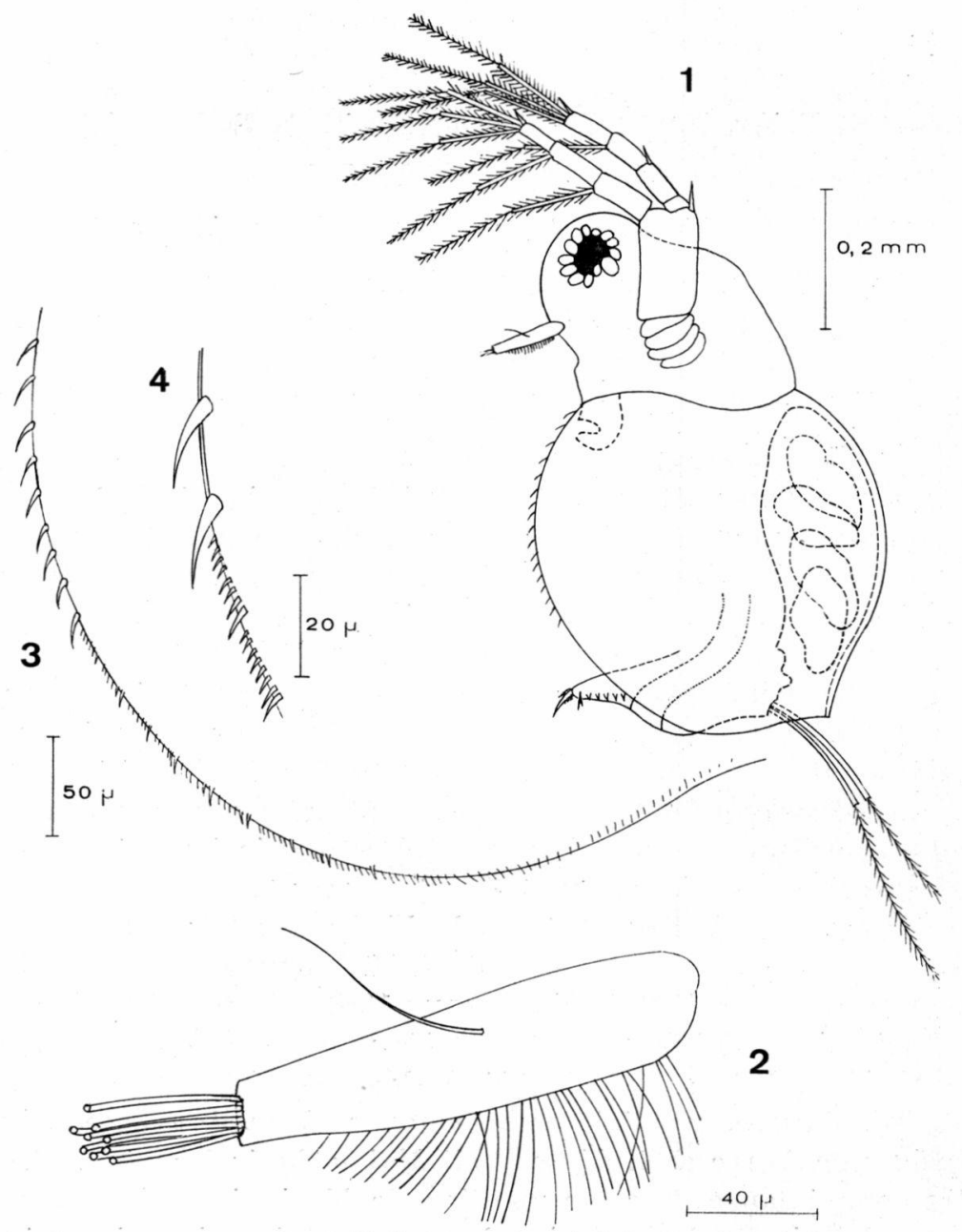

Fig. 1 à 4. - Moina dubia G. et R. - ㅇ parthénogénétique. 1 : 9 parthénogénétique. 2 : antennule gauche, face externe. 3 : marge libre, face interne, de la valve droite. 4 : détail de la ciliation de la même valve.

prenait une trentaine de $q$ parthénogénétiques (jeunes $\$ \%$ non ovigères, 우 gravides) et quelques + éphippiales.

Afin de permettre des comparaisons avec d'autres formes, nous donnons ci-dessous une brève description des caractères principaux des individus du lac de Rabastens, suivie d'un tableau indiquant les limites de variation des caractères mesurés. Ces mensurations ont été effectuées d'après les règles adoptées par H. GauthiER [1955] dans son important travail sur quelques espèces africaines de Moina. 
¡o parthénogénétiques (fig. 1). - Chez tous les exemplaires examinés, on note la présence d'un sinus frontal. L'antennule (fig. 2) est grêle et, dans tous les cas, porte sur le bord postérieur une rangée de cils longs et très fins. Elle est terminée par 9 filaments sensoriels mesurant à peu près $1 / 3$ de toute sa longueur.

Bord ventral des valves (fig. 3 et 4 ) garni dans la région antérieure de 14 à 17 soies spinuleuses, se poursuivant par 8 à 9 séries de 6 à 8 soies de taille croissante de l'avant vers l'arrière. Cette sériation s'atténue progressivement et la ciliation devient homogène et peu marquée dans la région tout à fait postérieure.

Le postabdomen ( fig. 5) porte de 4 à 5 processus ciliés latéraux à peu près 3 fois plus longs que larges. Ciliation de la région préanale représentée par de courtes soies disposées en rangées irrégulières et visibles seulement au fort grossissement.
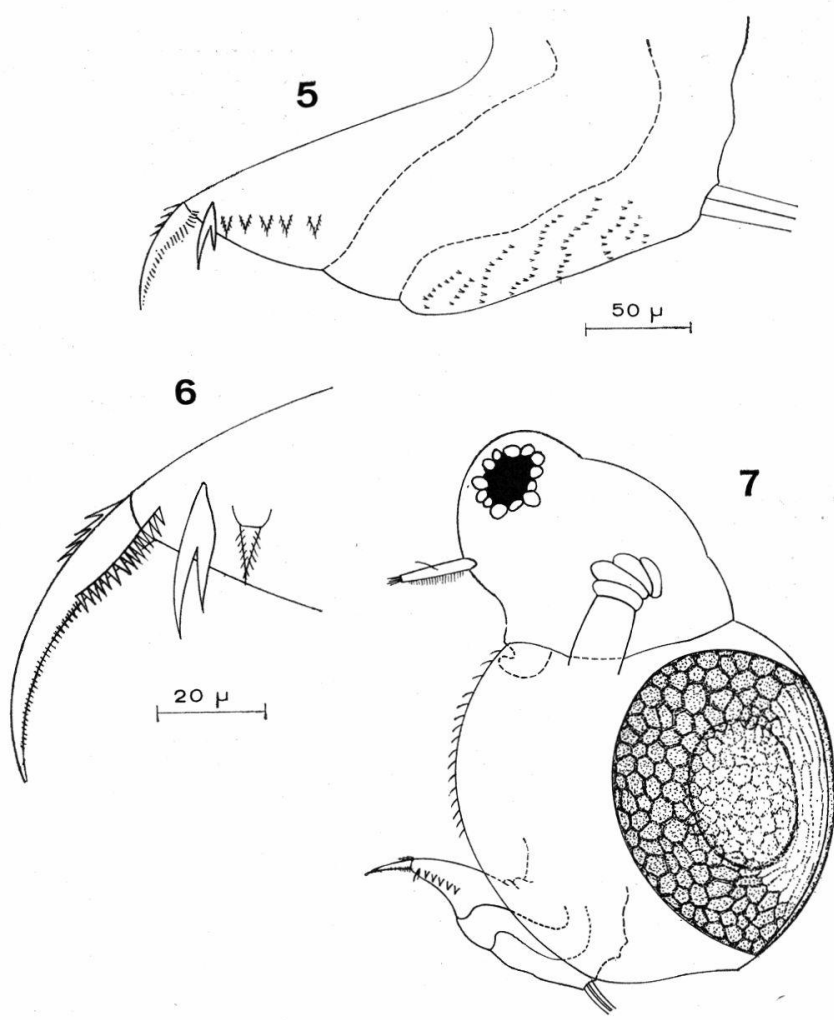

$0,2 \mathrm{~mm}$

FIG. 5 à 7. - Moina dubia G. et R. FIG. 5 et 6 : $ᄋ$ parthénogénétique. 5 : post-abdomen, vue latérale. 6 : griffe post-abdominale gauche, face externe, Fıc. 7 : 우 éphippiale. 
Les griffes postabdominales (fig. 6) ont un pseudo-peigne nettement défini, formé de 12 à 15 dents s'insérant sur une crête arquée et suivi d'une fine ciliation allant presque jusqu'à l'extrémité de la griffe. Près de l'insertion des griffes : 4 à 5 épines basales.

†o éphippiales (fig. 7). - Caractères morphologiques identiques à ceux des $\$ q$ parthénogénétiques.

L'éphippie est monoloculaire et a un seul œuf de durée. La réticulation, bien marquée sur le reste de l'éphippie, devient indistincte au niveau de la loge et le long de la crête dorsale. Nous n'avons pu, malheureusement, observer d'éphippies entièrement formées et détachées et, de ce fait, préciser comme le préconise H. Gauthier [1955, p. 51], la structure de la partie antérieure de l'éphippie vue de dos.

Les mesures effectuées sur les individus du lac de Rabastens donnent les chiffres suivants :

- Rapport de la longueur de la tête à la longueur des valves. ......................

- Rapport de la largeur à la longueur de l'antennule. $\quad 0,18$ à 0,23

- Rapport de la longueur de l'antennule à la longueur de la tête. . . .....................

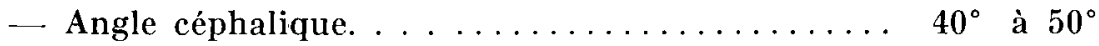

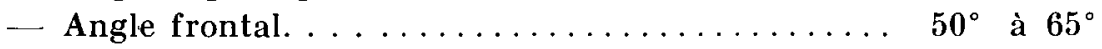

- Taille en $\mu \ldots \ldots \ldots \ldots \ldots \ldots \ldots \ldots \ldots 25$ à 850

Remarques systématiques et écologiques. - D'une manière générale, les exemplaires examinés correspondent à la diagnose de Moina dubia s. str. donnée par H. Gauthier [1955]. Ils en diffèrent cependant par l'affaiblissement de la réticulation sur les parois de la loge et au voisinage de la crête dorsale, rappelant en cela la forme de Leipzig [cf. W. Ramner 1931, p. 627]. Cette observation serait toutefois à revoir, du fait qu'elle porte comme nous l'avons dit, sur des éphippies incomplètement mûres et non détachées.

Nous avons tenté de retrouver Moina dubia mais une série de prélèvements effectués dans la station en août et septembre 1965 n'a donné aucun résultat. Il faut cependant signaler à ce sujet que, par suite des conditions atmosphériques particulièrement défavorables, le lac s'est maintenu à cette époque à des températures comprises entre $16^{\circ} \mathrm{C}$ et $21^{\circ} \mathrm{C}$. Or H. Gauthier [1955], à la suite de multiples observations faites dans la nature et expérimentalement, indique comme favorables au développement de l'espèce des températures de l'ordre de $22^{\circ} \mathrm{C}$ à $33^{\circ} \mathrm{C}$; les températures de $15^{\circ} \mathrm{C}$ à $22^{\circ} \mathrm{C}$ sont considérées par lui comme pathogènes froides. Ainsi, on peut penser que l'espèce n'a pas trouvé cette année-là de conditions thermiques favorables à son développement, ou qu'elle s'est développée de façon beaucoup plus précoce dans la saison. Il serait donc 
intéressant de retrouver cette population de Moina, afin de suivre son évolution dans la station même et tenter de préciser expérimentalement ses exigences thermiques.

\section{ON THE PRESEnCE OF MOINA DUbIa de Guerne et Richard, IN THE SOUTHWESTERN PART OF FRANCE}

[Cladocera, Daphniidae]

Record in Western Europe of Moina dubia G. \& R., a new species for the fauna of France. The principal characters of the French form are briefly described and figured.

UEBER DAS VORKOMMEN VON MOINA DUBIA DE GUERNE FT RICHARD, IN SUEDWESTEN VON FRANKREICH

[Cladoceren, Daphniidae]

Die Verfasserin erwähnt das Vorkommen von Moina dubia G. \& R., in Frankreich. Die wichtigsten Kennzeichen der französischen Moina dubia werden beschrieben.

\section{TRAVAUX CITES}

DADAY (E.). 1910. - Untersuchungen über die Süsswasser-Mikrofauna Deutsch Ost-Afrikas. Cladocera. Zoologica, 23 (59) : 120-158.

Ekman (S.). 1904. - Cladoceren und freilebende Copepoden aus Aegypten und dem Sudan, in Results of the Swed. Zool. Exped. to Egypt and White Nile. Uppsala, 1 (26) : 1-18.

Gauthier (H.). 1937. - Euphyllopodes et Cladocères continentaux récoltés par M. Monod au Sahara occidental et en Mauritanie. Bull. Soc. sc. nat. Maroc, 17 : 75-98.

Gauthier (H.). 1939. - Contribution à l'étude de la faune dulçaquicole de la région du Tchad et particulièrement des Branchiopodes et des Ostracodes. Bull. Inst. fr. Afr. Noire, 1 (1) : 110-244.

Gauthier (H.). 1955. - Essai sur la variabilité, l'écologie, le déterminisme du sexe et la reproduction de quelques Moina (Cladocères) récoltées en Afrique et là Madagascar. Alger : 1-249.

Guerne (J. de) et Richard (J.). 1892. - Cladocères et Copépodes d'eau douce des environs de Rufisque. Mém. Soc. Zool. Fr., 5 : 526-538.

GuRNey (R.). 1911. - On some freshwater Entomostraca from Egypt and the Soudan. Ann. Mag. nat. hist., sér. 8, $7: 25-33$.

GuRnEy (R.). 1927. - Some australian freshwater Entomostraca reared from dried mud. Proc. Zool. Soc. Lond., $1927: 59-79$.

Harding (J. P.). 1957. - Crustaca : Cladocera, in Exploration hydrobiologique du lac Tanganika. 3 (6) : 55-89.

Jenkin (P. M.). 1934. - Cladocera from the Rift Valley lakes in Kenya. Ann. Mag. nat. hist., ser. 10, 13 : 137-160; 281-308. 
LöFflER (H.). 1961. - Beitrage zur Kenntnis der Iranischen Binnengewässer. II. Regional limnologische Studie mit besonderer Berücksichtigung der Crustaceenfauna. Int. Rev. Hydrobiol., 46 (3) : 309-406.

LöfFlen (H.). 1963. - Ergebnisse der Zoologischen Nubien - Expedition 1962. XVIII. Zur Binnenwasserfauna einiger Kleingewässer und Brunnen im nördlichen Sudan. Ann. Naturhistor. Mus. Wien. 66 : 489-494.

Margalef (R.). 1961. - La vida en los charcos de aqua dulce de Nueve Esparta (Venezuela). Mem. Soc. Cienc. nat. La Salle, 21 (59) : 75-110.

Ramner (W.). 1931. --- Ein Vorkommen von Moina dubia de Guerne und Richard in Deutschland. Arch. f. Hydrobiol., 22 : 623-634.

Ramner (W.). 1933. - Zoologische Ergebnisse einer Reise nach Bonaire, Curaçao und Aruba im Jahre 1930. 4. Süsswasser und Brackwasser Phyllopoden von Bonaire. Zool. Jahrb. System., 64 : 357-368.

Ramner (W.). 1937. - Beitrag zur Cladocerenfauna von Java. Int. Rev. Hydrobiol., $35: 35-50$.

Steuer (A.). 1939. -. Variabilität und Verbreitung von Moina dubia G. R. Sitzungsb. Akad. Wissensch. Wien, 148 : 269-278.

Ueno (M.). 1938 a. - Cladocera fauna of Formosa. Bull. Biogeogr. Soc. Japan, 8 (8) : 121-132.

Ueno (M.). 1938 b. - Notes on the Cladocera of Dalai-Nor and its neighbouring waters. Annot. Zool. Jap., 17 (1) : 1-16.

Ueno (M.). 1939. - Manchurian freshwater Cladocera. Annot. Zool. Jap., 18 : $219-231$.

Weitaner (W.). 1898. - Ostafrikanische Cladoceren gesammelt von Herrn Dr. Stuhlmann 1888 und 1889. Mitt. Naturh. Mus. Hamburg, 15 : 135-144.

(Laboratoire de Zoologie, Faculté des Sciences, 118 , route de Narbonne, Toulouse.) 